\title{
SENSIBILIDAD AL CROMO: MICROBIOPRUEBAS CON LAS DIATOMEAS MARINAS Isochrysis galbana Parke Y Chaetoceros gracilis Schütt
}

\section{SENSIBILITY TO CHROMIUM: MICROBIOASSAY WITH MARINE DIATOM Isochrysis galbana Parke AND Chaetoceros gracilis Schütt}

\author{
Marianella Alayo ${ }^{1}$, José Iannacone ${ }^{2}$ y Anita Arrascue ${ }^{3}$
}

\section{Resumen}

En el Perú, las microalgas marinas Isochrysis galbana Parke y Chaetoceros gracilis Schütt, son utilizadas como alimentos de varias especies zoológicas de la cadena trófica marina; a su vez en condiciones de cultivo evita la utilización de la levadura de panificación, disminuyendo la acidificación del medio de cultivo y evitando así perjudicar a la acuicultura marina. El cromo es un contaminante de importancia antropogénica en el ecosistema acuático. En este trabajo se analiza el efecto del $\mathrm{Cr}^{+6}$ bajo la forma de $\mathrm{K}_{2} \mathrm{Cr}_{2} \mathrm{O}_{7}$ en las microbiopruebas de ecotoxicidad de inhibición con las microalgas marinas, I. galbana y $C$. gracilis usando un estimador de crecimiento poblacional específico para cuantificar la respuesta de toxicidad. Se realizó el bioensayo teniendo como resultado el análisis de sensibilidad al $\mathrm{K}_{2} \mathrm{Cr}_{2} \mathrm{O}_{7}$, productividad, costoeficiencia y su aplicación potencial como una herramienta para el monitoreo ambiental de rutina. Los valores de Concentración de Inhibición media $\left(\mathrm{CI}_{50}\right)$ entre $24 \mathrm{~h}$ hasta $120 \mathrm{~h}$ para I. galbana fueron: $11.55 \mathrm{mg} \mathrm{L}^{-1}\left(8.15\right.$ - 16.38) $(24 \mathrm{~h}) ; 14.14 \mathrm{mg} \mathrm{L}^{-1}(11.60-17.24)(48 \mathrm{~h}) ; 4.47 \mathrm{mg} \mathrm{L}^{-1}(3.24$ - 6.15) (72 h); $11.94 \mathrm{mg} \mathrm{L}^{-1}(8.14-17.49)(96 \mathrm{~h})$ y $19.20 \mathrm{mg} \mathrm{L}^{-1}$ (13.64- 27.26) (120 h). En cambio los valores de $\mathrm{CI}_{50}$ entre $24 \mathrm{~h}$ hasta $120 \mathrm{~h}$ para $C$. gracilis fueron: $9.21 \mathrm{mg} \mathrm{L}^{-1}(7.46-11.08)(24$ h); $8.13 \mathrm{mg} \mathrm{L}^{-1}\left(2.09\right.$ - 15.24) (48 h); $12.51 \mathrm{mg} \mathrm{L}^{-1}\left(6.80\right.$ - 20.04) $(72 \mathrm{~h})$; $12.17 \mathrm{mg} \mathrm{L}^{-1}(2.23-$ $34.32)(96 \mathrm{~h})$ y $13.34 \mathrm{mg} \mathrm{L}^{-1}(1.19-77.61)(120 \mathrm{~h})$. Los valores de $\mathrm{pH} \mathrm{y} \mathrm{T}^{\mathrm{o}}$ fueron evaluados a través de todo el bioensayo. Se compara la toxicidad del cromo con otras microbiopruebas acuáticas. Finalmente se analizan las perspectivas de empleo, en el Perú, de esta herramienta ecotoxicológica para la evaluación del riesgo ambiental del cromo.

Palabras claves: bioensayo, cromo, ecotoxicología, microalga marina.

\begin{abstract}
In Peru, marine microalgaes Isochrysis galbana Parke and Chaetoceros gracilis Schütt, are employed as food source for many zoological species of the marine trophic chain. During culture conditions they prevent the use of yeasts, diminishing the acidification of culture media and in that way, avoiding injuries to the marine aquaculture. Chromium is a pollutant of anthropogenic importance in the aquatic ecosystem. In this research, the effect of $\mathrm{Cr}^{+6}$ in form of $\mathrm{K}_{2} \mathrm{Cr}_{2} \mathrm{O}_{7}$ was analyzed in ecotoxicity inhibition microbioassays with marine microalgae, I. galbana and $C$. gracilis using an estimator of specific population growth to quantify ecotoxicity responses. Bioassays were performed providing results related to the sensibility to $\mathrm{K}_{2} \mathrm{Cr}_{2} \mathrm{O}_{7}$ analysis, productivity, cost-efficiency and their potential application as tools for routine environmental monitoring. The values of medial Concentration of Inhibition $\left(\mathrm{IC}_{50}\right)$ from $24 \mathrm{~h}$ to $120 \mathrm{~h}$ for $I$. galbana were: $11.55 \mathrm{mg} \mathrm{L}^{-1}\left(8.15\right.$ - 16.38) $(24 \mathrm{~h}) ; 14.14 \mathrm{mg} \mathrm{L}^{-1}(11.60-17.24)(48 \mathrm{~h}) ; 4.47 \mathrm{mg} \mathrm{L}^{-}$ ${ }^{1}$ (3.24 - 6.15) (72 h); $11.94 \mathrm{mg} \mathrm{L}^{-1}(8.14-17.49)(96 \mathrm{~h})$ and $19.20 \mathrm{mg} \mathrm{L}^{-1}$ (13.64- 27.26) (120 h). Conversely the values of $\mathrm{IC}_{50}$ from $24 \mathrm{~h}$ to $120 \mathrm{~h}$ for $C$. gracilis were: $9.21 \mathrm{mg} \mathrm{L}^{-1}(7.46-11.08)$ (24 h); $8.13 \mathrm{mg} \mathrm{L}^{-1}\left(2.09\right.$ - 15.24) (48 h); $12.51 \mathrm{mg} \mathrm{L}^{-1}\left(6.80\right.$ - 20.04) (72 h); $12.17 \mathrm{mg} \mathrm{L}^{-1}(2.23-$ 34.32) $(96 \mathrm{~h})$ and $13.34 \mathrm{mg} \mathrm{L}^{-1}(1.19-77.61)(120 \mathrm{~h})$. Temperature and $\mathrm{pH}$ values were evaluated in all bioassays. The toxicity of chromium was compared with other aquatic microbioassays. Finally, perspectives of employing these ecotoxicological tools to evaluate environmental risk assessment of chromium in Peru are analyzed.
\end{abstract}

Key words: bioassays, chromium, ecotoxicology, marine microalgae.

\section{Introducción}

Pruebas de ecotoxicidad con microalgas marinas han sido realizadas por numerosos investigadores (Lewis, 1993; Corradi \& Gorbi, 1993; Mallick \& Mohn, 2003). Para la selección de una microalga marina como herramienta ecotoxicológica deben conjugarse tanto aspectos biológicos como funcionales de laboratorio (Alayo \& Iannacone, 2000). Las ventajas relativas del uso de una microalga en particular dependen del cultivo, crecimiento, manipulación, sensibilidad y abundancia en la naturaleza (Lewis, 1993; Gorbi et al., 2001). Varias 
especies de microalgas marinas han sido propuestas como Thalassiosira spp. Dunaliella spp. e Isochrysis sp. (Pawlisz et al., 1997; Silva et al., 2001). No se encuentra, hasta el momento, una especie particular de diatomea marina que haya sido designada como organismo de bioensayo estandarizado (Cifuentes et al., 1998). El $\mathrm{Cr}^{6+}$ es un metal pesado capaz de producir una respuesta tóxica en la biota acuática, además es un químico de referencia para la estandarización de nuevos protocolos de bioensayos (APHA, 1995; Villaescusa et al., 1997; Whalley et al., 1999; Cervantes et al., 2001; Gorbi et al., 2002; Blackmore \& Wang, 2003; Rocchetta et al., 2003; Kobayashi \& Okamura, 2004; Viamajala et al., 2004; Zhu et al., 2004). Cifuentes et al. (1998), han evaluado microalgas marinas y dulceacuícolas como Chlamydomonas sp. Ehrenberg, Stichococcus bacillaris Nageli, Scenedesmus spinosus Chodat, Selenastrum capricornutum (=Raphidocephis subcapitata) Printz, Chlorella sp. Beijerinck, Ankistrodesmus sp. Corda, Dunaliella lateralis Pasher y Jahoda, Isochrysis galbana Parke y Dunaliella tertiolecta Butcher que fueron expuestas al $\mathrm{Cr}^{+6}$ bajo la forma de bicromato de potasio.

Las microalgas marinas Isochrysis galbana Parke y Chaetoceros gracilis Schütt son utilizadas como alimentos de varias especies zoológicas de la cadena trófica marina; a su vez en condiciones de cultivo evita la utilización de la levadura de panificación, disminuyendo la acidificación del medio de cultivo y evitando así perjudicar a la acuicultura marina (Otero et al., 1997; Aranda \& Suarez, 1998; Browne et al., 1998; Podemsky et al., 2001; Vera et al., 2001; Gibson, 2003; Mann \& Harding, 2003; Yap et al., 2004).

El objetivo de la presente investigación fue evaluar el efecto del $\mathrm{Cr}^{6+}$ en la prueba de inhibición de crecimiento de las microalgas marinas I. galbana y $C$. gracilis, analizando el efecto del $\mathrm{pH}$, temperatura, tiempo de exposición y punto final del ensayo, usando un estimador de crecimiento poblacional específico para cuantificar la respuesta de toxicidad [Concentración de Inhibición media, $\mathrm{CI}_{50}$ en $\mathrm{mg} \mathrm{L}^{-1}$ ] (Blaise et al., 1996). Además se comparó la toxicidad del cromo con otras especies de microalgas.

\section{Materiales y métodos}

Diatomeas Isochrysis galbana y Chaetoceros gracilis

Las cepas de I. galbana y $C$. gracilis fueron obtenidas del Cepario del Laboratorio de Microalgas del Área de Cultivos Marinos del Instituto del Mar del Perú (IMARPE). Ambas microalgas fueron sembradas inicialmente en Agar-agar en placas Petri y posteriormente transferidas a tubos de ensayo en fase líquida en un medio Guillard " $\mathrm{f} / 2$ " modificado con agua marina Ultravioleta (UV), filtrada a $0.22 \mu$ (Alayo \& Iannacone, 2000) con presencia de las sales necesarias, siguiendo la propuesta de la U.S.
Environmental Protection Agency (USEPA, 1978; Vera et al., 2001). Para los cultivos iniciales se utilizaron $8 \mathrm{~mL}$ de microalgas mezcladas con agua UV filtrada a $0.45 \mu$ bajo las siguientes condiciones: tubos fluorescentes de luz blanca fría colocados a ambos lados de la cámara de cultivo y a la temperatura ambiente de $23^{\circ} \mathrm{C} \pm 3^{\circ} \mathrm{C}$. Se realizaron las transferencias al medio fresco cada cuatro días con el fin de mantener un suministro constante de células en fase de crecimiento exponencial.

\section{Bicromato de potasio $\left(\mathrm{K}_{2} \mathrm{Cr}_{2} \mathbf{0}_{7}\right)$ y pruebas de} toxicidad

La solución madre a $1000 \mathrm{mg} \mathrm{L}^{-1}$ de $\mathrm{Cr}^{6+}$ se preparó a partir de $\mathrm{K}_{2} \mathrm{Cr}_{2} 0_{7}$ en agua destilada. Las pruebas de ecotoxicidad estáticas, sin aireación se iniciaron cuando el cultivo inicial de I. galbana y $C$. gracilis alcanzó una concentración de $30 \times 10^{4}$ células $\mathrm{ml}^{-1}$. Para I. galbana, las seis concentraciones de $\mathrm{Cr}$ empleadas fueron en orden creciente: $0.65 ; 1.25 ; 2.5$; $5 ; 10$ y $20 \mathrm{mg} \mathrm{L}^{-1}$. y para $C$. gracilis fueron en orden creciente: $2.5 ; 6.5 ; 11.5 ; 17.5 ; 24.5$ y $32.5 \mathrm{mg} \mathrm{L}^{-1}$. La estimación de la densidad celular se realizó mediante ocho conteos por concentración con la ayuda de un hemocitómetro. Las lecturas se realizaron diariamente entre $24 \mathrm{~h}$ y $120 \mathrm{~h}$ de exposición (Butterwick et al., 1982). En una de las concentraciones se evaluó exclusivamente el $\mathrm{pH}$ y la temperatura, al inicio y cada 24 h de exposición. La salinidad del agua de mar usada en los bioensayos fue de $35 \%$.

Tabla 1. Concentración de Inhibición media $\left(\mathrm{CI}_{50}\right)$ para Isochrysis galbana y Chaetoceros gracilis a 24, 48, 72, 96 y $120 \mathrm{~h}$ de exposición al $\mathrm{Cr}^{6+}$.

\begin{tabular}{ccccc}
\multicolumn{5}{l}{$\mathrm{CI}_{50}\left(\mathrm{mg} \mathrm{l}^{-1}\right)$} \\
\hline $\begin{array}{c}\text { Tiemp } \\
\text { os de } \\
\begin{array}{c}\text { Exposi } \\
\text { ción } \\
\text { (h) }\end{array}\end{array}$ & Isochrysis galbana & Sig. & Chaetoceros gracilis & Sig. \\
\hline 24 & $11.55(8.15-16.38)$ & $\mathrm{a}$ & $9.21(7.46-11.08)$ & \\
48 & $14.14(11.60-17.24)$ & $\mathrm{a}$ & $8.13(2.09-15.24)$ & $\mathrm{a}$ \\
72 & $4.47(3.24-6.15)$ & $\mathrm{b}$ & $12.51(6.80-20.04)$ & $\mathrm{a}$ \\
96 & $11.94(8.14-17.49)$ & $\mathrm{a}$ & $12.17(2.23-34.32)$ & $\mathrm{a}$ \\
120 & $19.20(13.64-27.26)$ & $\mathrm{a}$ & $13.34(1.19-77.61)$ & $\mathrm{a}$ \\
\hline
\end{tabular}

Letras minúsculas iguales en una misma columna indica que los promedios son estadísticamente iguales. Valores de Tukey a un nivel de significancia de 0.05 .

Sig. $=$ Significancia o probabilidad.

\section{Análisis estadístico}

Las pruebas de toxicidad se realizaron en tres repeticiones con seis concentraciones nominales más el control, en cada repetición se realizaron cuatro lecturas de densidad microalgal (células $\mathrm{ml}^{-1}$ ), en un Diseño en Bloques Completamente Aleatorizado (DBCA). La eficacia de los tratamientos se evaluó a través de un ANDEVA. 
Tabla 2. Valores comparativos de $\mathrm{pH}$ y temperatura del agua de mar entre concentraciones ensayadas para Isochrysis galbana y Chaetoceros gracilis.

\begin{tabular}{|c|c|c|c|c|c|c|c|c|c|}
\hline \multicolumn{5}{|c|}{ Isochrysis galbana } & \multicolumn{5}{|c|}{ Chaetoceros gracilis } \\
\hline $\begin{array}{l}\text { Concentraciones } \\
\left(\mathrm{mg} \mathrm{L}^{-1}\right)\end{array}$ & $\mathrm{pH}$ & Sig. & Temperatura & Sig. & $\begin{array}{l}\text { Concentraciones } \\
\left(\mathrm{mg} \mathrm{L}^{-1}\right)\end{array}$ & $\mathrm{pH}$ & Sig. & $\begin{array}{c}\text { Temperatur } \\
\mathrm{a}\end{array}$ & $\begin{array}{l}\text { Si } \\
\text { g. }\end{array}$ \\
\hline 0 & $8.55 \pm 0,18$ & $\mathrm{a}$ & $\begin{array}{c}28.96 \pm \\
0.77\end{array}$ & A & 0 & $\begin{array}{c}8.74 \pm \\
0.23\end{array}$ & $\mathrm{ab}$ & $\begin{array}{c}27.69 \pm \\
0.99\end{array}$ & $\mathrm{a}$ \\
\hline 0.65 & $\begin{array}{c}8.76 \pm \\
0.22\end{array}$ & $\mathrm{a}$ & $\begin{array}{c}29.10 \pm \\
0.77\end{array}$ & A & 2.5 & $\begin{array}{c}8.63 \pm \\
0.17\end{array}$ & $\mathrm{ab}$ & $\begin{array}{c}27.45 \pm \\
1.10\end{array}$ & $\mathrm{a}$ \\
\hline 1.25 & $\begin{array}{c}9.06 \pm \\
0.46\end{array}$ & $\mathrm{a}$ & $\begin{array}{c}29.33 \pm \\
0.64\end{array}$ & $\mathrm{a}$ & 6.5 & $\begin{array}{c}8.81 \pm \\
0.32\end{array}$ & $\mathrm{ab}$ & $\begin{array}{c}28.03 \pm \\
0.85\end{array}$ & $\mathrm{a}$ \\
\hline 2.5 & $\begin{array}{c}8.66 \pm \\
0.41\end{array}$ & $\mathrm{a}$ & $\begin{array}{c}29.55 \pm \\
0.70\end{array}$ & a & 11.5 & $\begin{array}{c}8.88 \pm \\
0.47\end{array}$ & $\mathrm{~b}$ & $\begin{array}{c}28.03 \pm \\
0.76\end{array}$ & a \\
\hline 5 & $\begin{array}{c}8.53 \pm \\
0.18\end{array}$ & $\mathrm{a}$ & $\begin{array}{c}28.83 \pm \\
0.81\end{array}$ & a & 17.5 & $\begin{array}{c}8.49 \pm \\
0.21\end{array}$ & $a b$ & $\begin{array}{c}27.91 \pm \\
0.88\end{array}$ & $\mathrm{a}$ \\
\hline 10 & $\begin{array}{c}9.01 \pm \\
0.46\end{array}$ & $\mathrm{a}$ & $\begin{array}{c}28.18 \pm \\
1.33\end{array}$ & $\mathrm{a}$ & 24.5 & $\begin{array}{c}8.38 \pm \\
0.21\end{array}$ & $a b$ & $\begin{array}{c}27.91 \pm \\
0.94\end{array}$ & $\mathrm{a}$ \\
\hline 20 & $\begin{array}{c}8.75 \pm \\
0.57\end{array}$ & $\mathrm{a}$ & $\begin{array}{c}28.11 \pm \\
0.73\end{array}$ & $\mathrm{a}$ & 32.5 & $\begin{array}{c}8.32 \pm \\
0.27\end{array}$ & $\mathrm{a}$ & $\begin{array}{c}27.60 \pm \\
0.94\end{array}$ & $\mathrm{a}$ \\
\hline $\mathrm{F}$ & 1.71 & & 2.47 & & $\mathrm{~F}$ & 3.34 & & 0.35 & \\
\hline Sig. & 0.23 & & 0.07 & & Sig. & $0.01 * *$ & & 0.90 & \\
\hline
\end{tabular}

Letras minúsculas iguales en una misma columna indica que los promedios son estadísticamente iguales.

Valores de Tukey a un nivel de significancia de 0.05 .

$\mathrm{F}=$ Estadístico de Fisher.

Sig. $=$ Significancia o probabilidad.

Tabla 3. Valores comparativos de $\mathrm{pH}$ y temperatura del agua de mar entre periodos de exposición para Isochrysis galbana y Chaetoceros gracilis.

\begin{tabular}{|c|c|c|c|c|c|c|c|c|c|}
\hline \multicolumn{5}{|c|}{ Isochrysis galbana } & \multicolumn{5}{|c|}{ Chaetoceros gracilis } \\
\hline $\begin{array}{l}\text { Periodos de } \\
\text { Exposición } \\
\text { (h) }\end{array}$ & $\mathrm{pH}$ & Sig. & Temperatura & Sig. & $\begin{array}{c}\text { Periodos de } \\
\text { Exposición } \\
\text { (h) }\end{array}$ & $\mathrm{pH}$ & Sig. & Temperatura & Sig. \\
\hline 0 & $\begin{array}{c}8.84 \pm \\
0.48\end{array}$ & $\mathrm{a}$ & $\begin{array}{c}28.87 \pm \\
0.83\end{array}$ & $\mathrm{a}$ & 0 & $\begin{array}{l}8.13 \pm \\
0.20\end{array}$ & & $29.15 \pm 0.26$ & d \\
\hline 24 & $\begin{array}{c}8.80 \pm \\
0.43\end{array}$ & $\mathrm{a}$ & $\begin{array}{c}28.91 \pm \\
0.95\end{array}$ & $\mathrm{a}$ & 24 & $\begin{array}{l}8.50 \pm \\
0.11\end{array}$ & $a b$ & $28.21 \pm 0.15$ & $\mathrm{c}$ \\
\hline 48 & $\begin{array}{c}8.78 \pm \\
0.55\end{array}$ & $\mathrm{a}$ & $\begin{array}{c}29.21 \pm \\
0.55\end{array}$ & $\mathrm{a}$ & 48 & $\begin{array}{l}8.75 \pm \\
0.26\end{array}$ & $a b$ & $27.85 \pm 0.41$ & $\mathrm{c}$ \\
\hline 72 & $\begin{array}{c}8.58 \pm \\
0.15\end{array}$ & $\mathrm{a}$ & $\begin{array}{c}28.18 \pm \\
1.48\end{array}$ & $\mathrm{a}$ & 72 & $\begin{array}{l}8.74 \pm \\
0.24\end{array}$ & $a b$ & $27.14 \pm 0.55$ & b \\
\hline 96 & $\begin{array}{c}8.81 \pm \\
0.46\end{array}$ & $\mathrm{a}$ & $\begin{array}{c}28.87 \pm \\
0.78\end{array}$ & $\mathrm{a}$ & 96 & $\begin{array}{l}8.72 \pm \\
0.22\end{array}$ & $\mathrm{~b}$ & $26.55 \pm 0.31$ & $\mathrm{a}$ \\
\hline 120 & $\begin{array}{c}8.76 \pm \\
0.32\end{array}$ & $\mathrm{a}$ & $\begin{array}{c}29.15 \pm \\
0.66\end{array}$ & $\mathrm{a}$ & 120 & $\begin{array}{l}8.79 \pm \\
0.38\end{array}$ & $a b$ & $27.91 \pm 0.88$ & $\mathrm{c}$ \\
\hline $\mathrm{F}$ & 0.34 & & 1.08 & & $\mathrm{~F}$ & 7,11 & & 47.28 & \\
\hline $\mathrm{P}$ & 0.88 & & 0.38 & & $\mathrm{P}$ & $0.00 * * *$ & & $0.00 * * *$ & \\
\hline
\end{tabular}

Letras minúsculas iguales en una misma columna indica que los promedios son estadísticamente iguales. Valores de Tukey a un nivel de significancia de 0.05 .

$\mathrm{F}=$ Estadístico de Fisher.

Sig. = Significancia o probabilidad.

La Concentración de Inhibición media $\left(\mathrm{CI}_{50}\right)$ con sus límites de confianza al $95 \%$ se calcularon usando un programa computarizado de la EPA (USEPA, 1993). Se determinó mediante el ANDEVA si existían diferencias entre los valores de $\mathrm{pH}$, temperatura y $\mathrm{CI}_{50}$ entre los cinco periodos de exposición. Además, se usó el ANDEVA para determinar si existían diferencias entre concentraciones ensayadas para cada especie de diatomea. El pH y la temperatura fueron comparados separadamente entre las dos especies de 
diatomeas mediante la prueba de $t$ de Student. Para el cálculo de todas las pruebas estadísticas inferenciales se usó el paquete estadístico SPSS versión 9.0.

\section{Resultados}

La Tabla 1 muestra los valores de $\mathrm{CI}_{50}$ con sus respectivos límites de confianza para los cinco periodos de exposición en I. galbana y en C. gracilis. Debería esperarse un incremento de la toxicidad del $\mathrm{Cr}^{6+}$ al aumentar el tiempo de exposición. Sin embargo, para ambas especies de diatomeas no se encontró este comportamiento (Tabla 1). En $I$. galbana el $\mathrm{pH}$ y la temperatura no variaron significativamente entre las concentraciones ensayadas y entre los periodos de exposición del bioensayo (Tabla 2 y 3 ). En contraste para $C$. gracilis, el $\mathrm{pH}$ y la temperatura variaron entre los tiempos de exposición, y solo la temperatura entre las seis concentraciones evaluadas (Tablas 2 y 3 ). $\quad \mathrm{La}$ comparación de los límites de confianza y de los resultados de la prueba de Tukey entre los cinco períodos de exposición de $24 \mathrm{~h}$ a $120 \mathrm{~h}$, muestra que no existen diferencias significativas en los valores de $\mathrm{CI}_{50}$ para $C$. gracilis; en cambio I. galbana mostró diferencias en el valores de $\mathrm{CI}_{50}$ entre las $72 \mathrm{~h}$ de exposición y los restantes tiempos de exposición. No existieron diferencias significativas entre los valores de $\mathrm{pH}$ del agua de mar en ambas especies de diatomeas marinas $(\mathrm{t}=1.75 ; \mathrm{P}=0.08)$; en cambio la temperatura de exposición de I. galbana fue ligeramente mayor que la de $C$. gracilis $(\mathrm{t}=5.52 ; \mathrm{P}=$ $0.00)$. La Tabla 4 nos muestra los valores comparativos de $\mathrm{CI}_{50}$ del $\mathrm{Cr}^{6+}$ con varias especies de microalgas.

\section{Discusión}

Procedimientos de pruebas estándar para conducir pruebas de toxicidad con microalgas marinas han sido publicados por numerosas organizaciones como la Agencia de Protección Ambiental (USEPA, 1978), la Asociación Americana de Salud Pública (APHA, 1995), la Sociedad Americana para la Evaluación de Materiales (ASTM, 1990) y la Organización Internacional para la Cooperación Económica y Desarrollo (OECD, 1984). Con estos métodos estándares, que son muy similares, se determina el efecto tóxico en un crecimiento algal rápido en un medio enriquecido en nutrientes por 3-4 días (Lewis, 1993). La biomasa es medida diariamente o solamente al final de la prueba, el resultado es expresado como una función de la reducción en el crecimiento o biomasa (Janssen, 1998). En nuestro caso se siguió la metodología propuesta por la USEPA (1978), utilizando el método estándar de conteo bajo el microscopio con lecturas diarias y al final de la prueba a 96 h- 120 h de exposición. Sin embargo, a las 120 h de exposición se observa una caída en el crecimiento de la biomasa algal para ambas especies en términos de $\mathrm{CI}_{50}$ (Tabla 1). El control en ambas especies de microalgas mostró una caída en el crecimiento algal a $120 \mathrm{~h}$ de exposición. Además, en lugar de usar matraces Erlenmeyer de 125-250 ml de capacidad, se usaron tubos de ensayo de $12.15 \mathrm{ml}$. No se ha podido determinar si el pequeño volumen de $12.15 \mathrm{ml}$ empleado por tubo influyó en la caída en el crecimiento algal para ambas diatomeas en la microbioprueba. Sin embargo, Alayo \& Iannacone (2000) encontraron para Skeletonema costatum (Greville) Cleve, una caída en el crecimiento algal a $120 \mathrm{~h}$ de exposición y empleando tubo de igual tamaño.

Nylhom \& Källqvist (1989) señalan que cualquiera sea el método utilizado para determinar biomasa algal, se tiene que cumplir con la especificidad, facilidad manipulativa, precisión y límite de detección. El conteo bajo el microscopio es el método estándar que permite realizar calibraciones y comparaciones sistemáticas, aunque consume mucho tiempo. Debido a esto, decidimos usar el conteo del número de células $\mathrm{mL}^{-1}$ para C. gracilis y para I. galbana.

Janssen (1998) señala que varios métodos han sido descritos usando como puntos finales de prueba la medición de la fotosíntesis por asimilación de $\mathrm{C}^{14}$, consumo de oxígeno, fluorescencia clorofiliana in vivo. Otros métodos, usan como criterio la ingesta de nutrientes o de inhibición enzimática. Las investigaciones tienen el objetivo de incrementar la simplicidad y la eficiencia en el costo de las pruebas algales (Iannacone et al., 1997; Alayo \& Iannacone, 2000; Iannacone et al., 2001). Estos procedimientos incluyen la aplicación de la citometría en flujo, técnicas en microplacas e inmovilización algal. Una revisión de las pruebas alternativas a pequeña escala de bioensayos con algas es dada por Blaise et al. (1996). Willemsen et al. (1995) concluyó al comparar las pruebas microalgales realizadas con las bacterias e invertebrados, que las microalgas son muy sensibles a los metales, pero no muy sensibles a los compuestos orgánicos, como los plaguicidas (Iannacone \& Gutierrez, 1999). Lewis (1993, 1995) señala que la $\mathrm{CI}_{50}$ obtenida para un mismo tóxico y especie microalgal pueden variar mucho entre diferentes laboratorios, pudiendo esto minimizarse si se realiza bajo condiciones controladas. Así, las condiciones estandarizadas para la realización de los bioensayos con C. gracilis y con I. galbana se muestran en la Tabla 5.

La capacidad del cromo de inhibir la fotosíntesis ha sido reportada previamente para algunas algas. Los efectos dependen tanto de la naturaleza del organismo (tamaño celular, estructura de la pared celular, reacciones redox causadas por exudados o enzimas) y de la duración de la exposición (Chen et al., 2003; Pinto et al., 2003; Zayed \& Ferry, 2003). 
Tabla 4. Valores comparativos de $\mathrm{CI}_{50}$ con microalgas expuestas al $\mathrm{Cr}^{6+}$ a diferentes puntos finales de exposición.

\begin{tabular}{|c|c|c|c|c|c|c|}
\hline Especie & Hábitat & $\begin{array}{c}\text { Temperatura } \\
\left({ }^{\circ} \mathrm{C}\right) \\
\end{array}$ & $\mathbf{p H}$ & Efecto & $\operatorname{ug} L^{-1}$ & $\begin{array}{c}\text { Referencia } \\
\text { bibliográfica }\end{array}$ \\
\hline Scenedesmus sp. & $\mathrm{D}$ & 22 & 7.4 & $\mathrm{CI}_{50}$ a $96 \mathrm{~h}$ & 100000 & Pawlisz et al. (1997) \\
\hline Scenedesmus sp. & $\mathrm{D}$ & 22 & ni & $\mathrm{CI}_{50}$ a $96 \mathrm{~h}$ & 900 & Cifuentes et al., (1998) \\
\hline Selenastrum capricornutum Printz & $\mathrm{D}$ & 22 & ni & $\mathrm{CI}_{50}$ a $96 \mathrm{~h}$ & 2800 & Cifuentes et al. (1998) \\
\hline $\begin{array}{c}\text { Skeletonema costatum (Greville) } \\
\text { Cleve }\end{array}$ & M & 22 & ni & $\mathrm{CI}_{50}$ a $96 \mathrm{~h}$ & 2500 & Kusk \& Nyholm (1992) \\
\hline $\begin{array}{c}\text { Skeletonema costatum (Greville) } \\
\text { Cleve }\end{array}$ & M & 15 & 8.5 & $\mathrm{CI}_{50}$ a $96 \mathrm{~h}$ & 14700 & Cowgill et al. (1989) \\
\hline Dunaliella bioculata Butcher & M & 15 & ni & $\mathrm{CI}_{50}$ a $96 \mathrm{~h}$ & 77000 & Kusk \& Nyholm (1992) \\
\hline Dunaliella lateralis Butcher & M & 22 & ni & $\mathrm{CI}_{50}$ a $96 \mathrm{~h}$ & 3430 & Cifuentes et al. (1998) \\
\hline Chlorella sp. & $\mathrm{D}$ & 22 & ni & $\mathrm{CI}_{50}$ a $96 \mathrm{~h}$ & 800 & Cifuentes et al. (1998) \\
\hline Isochrysis galbana Parke & M & 22 & ni & $\mathrm{CI}_{50}$ a $96 \mathrm{~h}$ & 4300 & Cifuentes et al. (1998) \\
\hline Chlorella protothecoides Krüg & $\mathrm{D}$ & ni & ni & $\mathrm{CI}_{50}$ a $96 \mathrm{~h}$ & 4960 & Chen et al. (2003) \\
\hline Synechococcus sp. & $\mathrm{D}$ & ni & ni & $\mathrm{CI}_{50}$ a $96 \mathrm{~h}$ & 6500 & Chen et al. (2003) \\
\hline Spirulina maxina Setch \& Garner & $\mathrm{D}$ & ni & ni & $\mathrm{CI}_{50}$ a $96 \mathrm{~h}$ & 11160 & Chen et al. (2003) \\
\hline Spirulina platensis (Norst.) Geitler & $\mathrm{D}$ & ni & ni & $\mathrm{CI}_{50}$ a $96 \mathrm{~h}$ & 11740 & Chen et al. (2003) \\
\hline Selenastrum capricornutum Printz & $\mathrm{D}$ & ni & ni & $\mathrm{CI}_{50}$ a $96 \mathrm{~h}$ & 12430 & Chen et al. (2003) \\
\hline $\begin{array}{c}\text { Scenedesmus quadricauda (Turp.) } \\
\text { Bréb. }\end{array}$ & $\mathrm{D}$ & ni & ni & $\mathrm{CI}_{50}$ a $96 \mathrm{~h}$ & 20890 & Chen et al. (2003) \\
\hline $\begin{array}{c}\text { Skeletonema costatum (Greville) } \\
\text { Cleve }\end{array}$ & M & 27 & 8.4 & $\mathrm{CI}_{50}$ a $96 \mathrm{~h}$ & $122-273$ & $\begin{array}{l}\text { Alayo \& Iannacone } \\
\text { (2000) }\end{array}$ \\
\hline Chaetoceros gracilis Schütt & M & 27.9 & 8.7 & $\begin{array}{l}\mathrm{CI}_{50} \mathrm{a} \\
120 \mathrm{~h}\end{array}$ & 13340 & Original \\
\hline Chaetoceros gracilis Schütt & M & 26.5 & 8.7 & $\mathrm{CI}_{50}$ a $96 \mathrm{~h}$ & 12170 & Original \\
\hline Chaetoceros gracilis Schütt & M & 27.1 & 8.7 & $\mathrm{CI}_{50}$ a $72 \mathrm{~h}$ & 12510 & Original \\
\hline Chaetoceros gracilis Schütt & M & 27.8 & 8.7 & $\mathrm{CI}_{50}$ a $48 \mathrm{~h}$ & 8130 & Original \\
\hline Chaetoceros gracilis Schütt & M & 28.2 & 8.5 & $\mathrm{CI}_{50}$ a $24 \mathrm{~h}$ & 9210 & Original \\
\hline Isochrysis galbana Parke & M & 29.1 & 8.7 & $\begin{array}{l}\mathrm{CI}_{50} \mathrm{a} \\
120 \mathrm{~h}\end{array}$ & 19200 & Original \\
\hline Isochrysis galbana Parke & M & 28.8 & 8.8 & $\mathrm{CI}_{50}$ a $96 \mathrm{~h}$ & 11940 & Original \\
\hline Isochrysis galbana Parke & M & 28.1 & 8.5 & $\mathrm{CI}_{50}$ a $72 \mathrm{~h}$ & 4470 & Original \\
\hline Isochrysis galbana Parke & M & 29.2 & 8.7 & $\mathrm{CI}_{50}$ a $48 \mathrm{~h}$ & 14140 & Original \\
\hline Isochrysis galbana Parke & $\mathrm{M}$ & 28.9 & 8.8 & $\mathrm{CI}_{50}$ a $24 \mathrm{~h}$ & 11550 & Original \\
\hline
\end{tabular}

$\overline{\mathrm{D}}=$ Dulceacuícola; $\mathrm{M}=$ Marino; $\mathrm{ni}=$ no indicado.

Las microalgas como Isochrysis sp., Thalassiosira mariae-leburiae Cleve, Pavlova lutheri (Droop) Green y I. galbana presentan $\mathrm{CI}_{50 \mathrm{~s}}$ al $\mathrm{Cr}^{6+}$ de $1300 \mu \mathrm{g} \mathrm{L}^{-1}$, $900 \mu \mathrm{g} \mathrm{L} \mathrm{L}{ }^{-1}, \quad 1 \quad 700 \mu \mathrm{gL}^{-1}$ y $4 \quad 300 \mu \mathrm{g} \quad \mathrm{L}^{-1}$ respectivamente, y muestran valores más bajos que los encontrados en nuestro trabajo (Tablas 1 y 4). La microalga Euglena gracilis Klebs es considerada como un modelo intermedio entre los sistemas bacteriales y los modelos animales; se ha observado que el cromo no produce un efecto mutagénico, pero provoca efectos adversos en la curva de crecimiento microalgal (Gajdosova \& Reichrtova, 1996). Wong \& Chang (1991) indican que el Cr provoca efectos en el crecimiento, la fotosíntesis y en la síntesis de clorofila A del alga Chlorella protothecoides Krüg ( $C$. pyrenoidosa), y que actúa sinérgicamente con el $\mathrm{Cu}$ y el $\mathrm{Pb}$.

El ensayo con estas microalgas son fácilmente reproducibles y de bajo costo, pudiéndose llevar a cabo en un laboratorio de escasos recursos económicos. Este bioensayo puede usarse como una 
herramienta alternativa para el monitoreo ambiental de rutina (Lewis, 1993).

Tabla 5. Condiciones y criterios de aceptabilidad de la prueba de toxicidad con Isochrysis galbana y Chaetoceros gracilis.

\begin{tabular}{ll|} 
Tipo de bioensayo & $:$ Estático. \\
Tiempo de exposición & $: 24,48,72,96$ y $120 \mathrm{~h}$. \\
Temperatura & $: 26^{\circ} \mathrm{C}$ a $29^{\circ} \mathrm{C}$. \\
Calidad de luz & $:$ Iluminación con fluorescente \\
& de luz blanca. \\
Fotoperiodo & $: 24 \mathrm{~h} \mathrm{luz.}$ \\
Tamaño de envase & $: 12.5 \mathrm{ml}$. \\
Volumen de solución & $: 8 \mathrm{ml}$. \\
Edad de organismos & $:$ células en fase exponencial $<$ \\
& $72 \mathrm{~h}$. \\
$\mathrm{N}^{\circ}$ réplicas por concentración & $: 2$. \\
$\mathrm{N}^{\circ}$ de concentraciones más & $: 7$. \\
control & \\
Aireación & $:$ Ausente. \\
Tiempo de observación en la & $:$ conteo en hemocitómetro para \\
placa de conteo & el cálculo de $\mathrm{N}^{\circ}$ de células $\mathrm{ml}^{-1}$. \\
Respuesta Subletal & $:$ disminución de la biomasa \\
& microalgal. \\
Criterio de aceptabilidad & $:$ ajustabilidad de la curva de \\
\end{tabular}

\section{Conclusiones}

Ambas especies de microalgas marinas I. galbana y C. gracilis presentan similar sensibilidad al $\mathrm{Cr}$, y por ende son adecuadas como microbiopruebas de ecotoxicidad. Sin embargo, la menor variabilidad en I. galbana del $\mathrm{pH}$ y la temperatura entre las concentraciones ensayadas y entre los periodos de exposición, proporcionan ventajas comparativas y recomiendan a esta especie para su empleo en bioensayos a $24 \mathrm{~h}$ de exposición (fase de crecimiento exponencial) y con un valor de $\mathrm{CI}_{50}$ como punto final.

\section{Literatura citada}

Alayo M. \& Iannacone J. 2000. La microalga marina Skeletonema costatum (Greville) Cleve, como bioensayo alternativo para la evaluación del cromo. Wiñay Yachay. 4(2): 69-75.

APHA. (American Public Health Association), AWWA (American Water Works Association), WPCF (Water Pollution Control Federation). 1995. Standard methods for examination of water and wastewater. $19^{\text {th }}$. Ed. American Health Association. Washington, D.C.

Aranda A.D. \& Suarez P. 1998. Overview of diets used in larviculture of three caribbean conchs: Queen conch Strombus gigas, Milk conch Strombus costatus and Fighting conch Strombus pugilis. Aquaculture. 167: 163-178.

ASTM (American Society for Testing and Materials). 1990. Standard guide for conducting static 96h toxicity tests with microalgae, E1218-90. ASTM, Philadelphia.

Blackmore G. \& Wang W.X. 2003. Inter-population differences in $\mathrm{Cd}, \mathrm{Cr}$, Se, and $\mathrm{Zn}$ accumulation by the green mussel Perna viridis acclimated at different salinities. Aquatic Toxicology. 62: 205218.

Blaise C., Férard J.F. \& Vasseur P. 1996. Microplate toxicity tests with microalgae: a review. En: Microscale toxicology, advances, techniques and practice, Wells, P.G., Lee K., Blaise, C. (Eds.), CRC Publishers.

Browme K.A., Tamburri M.N. \& Zimmer-Faust R.K. 1998. Modelling quantitative structure-activity relationships between animal behavior and environmental signal molecule. The Journal of Experimental Biology. 201: 245-258.

Butterwick C., Heaney S.I. \& Talling J.F. 1982. A comparison of eight methods for estimating the biomass and growth of planktonic algae. British Phycology Journal. 17: 69-79.

Cervantes C., Campos-Garcia J., Devars S., GutierrezCorona F., Loza-Tavera H., Torres-Guzman J.C. \& Moreno-Sanchez R. 2001. Interactions of chromium with microorganisms and plants. FEMS Microbiology Review. 25: 335-347.

Chen H., Pan G., Yan H. \& Qin Y. 2003. Toxic effects of hexavalent chromium on the growth of the bluegreen microalgae [Artículo en Chino]. Huan Jing Ke Xue. 24: 13-18.

Cifuentes A.S., Silva J., Bay-Schmith E. \& Larrain A. 1998. Selección de cepas de microalgas para ser utilizadas en bioensayos de toxicidad. Rev. Gayana Oceanol. 6: 1-9.

Corradi M.G. \& Gorbi G. 1993. Chromium toxicity on two linked trophic levels. II. Morphophysiological effects on Scenedesmus acutus. Ecotoxicology and Environmental Safety. 25: 72-78.

Cowgill U., Milazzo D. \& Landenber B. 1989. Toxicity of nine benchmark chemicals to Skeletonema costatum, a marine diatom. Environmental Toxicology and Chemistry. 8: 451455.

Gajdosova J. \& Reichrtova E. 1996. Different growth response of Euglena gracilis to $\mathrm{Hg}, \mathrm{Cd}, \mathrm{Cr}$ and $\mathrm{Ni}$ compounds. Analysis and Bioanalysis Chemistry. 354: 641-642.

Gorbi G., Corradi M.G., Invidia M. \& Bassi M. 2001 Light intensity influences chromium bioaccumulation and toxicity in Scenedesmus acutus (Chlorophyceae). Ecotoxicology and Environmental Safety. 48: 36-42.

Gorbi G., Corradi M.G., Invidia M., Rivara L. \& Bassi M. 2002. Is $\mathrm{Cr}(\mathrm{VI})$ toxicity to Daphnia magna modified by food availability or algal exudates? The hypothesis of a specific chromium/algae/exudates interaction. Water Research. 36: 1917-1926.

Gibson G.D. 2003. Larval development and metamorphosis in Pleurobranchaea maculate, with a review of development in the Notaspidea (Opisthobranchia). Biological Bulletin. 205: 121132. 
Iannacone J. \& Gutierrez A. 1999. Ecotoxicidad de los agroquímicos lindano y clorpirifos sobre el nematodo Panagrellus, la microalga Chlorella y el ensayo con Allium. Agricultura técnica (Chile). 59: 85-95.

Iannacone J., Gutierrez A.I. \& Vargas R.N. 1997. Ecotoxicidad de la cuenca alta del río Rímac (Tamboraque y Perubar) utilizando al nematodo Panagrellus redivivus y a la microalga Chlorella vulgaris. Hipótesis (Perú). 5: 38-45.

Iannacone J., Alvariño L. \& Arrascue A. 2001. Inhibición por metales pesados en el ensayo ecotoxicológico con la microalga Chlorella vulgaris. Biota. 100: 138-144.

Janssen C. 1998. Alternative assay for routine toxicity assessments: a review. En: Ecotoxicology. G. Schüürmann \& B. Markert (Eds.). John Wiley \& Sons. Chapter. 26: 813-839.

Kobayashi N. \& Okamura H. 2004. Effects of heavy metals on sea urchin embryo development. 1. Tracing the cause by the effects. Chemosphere. 55: 1403-1412.

Kusk K. \& Nyholm N. 1992. Toxic effects of chlorinated compounds and potassium dichromate on growth rate and photosynthesis of marine phytoplankton. Chemosphere. 25: 875-886.

Lewis M.A. 1993. Freshwater primary producers. En: Handbook of Ecotoxicology. Calow, P. (ed.). Blackwell Scientific Publ. U.K. : 28-51.

Lewis M.A. 1995. Use of freshwater plants for phytotoxicity testing: a review. Environmental Pollution. 87: 319-336.

Mallick N. \& Mohn F.H. 2003. Use of chlorophyll fluorescence in metal-stress research: a case study with the green microalga Scenedesmus. Ecotoxicology and Environmental Safety. 55: 6469.

Mann R. \& Harding J.M. 2003. Salinity tolerance of larval Rapana venosa: implications for dispersal and establishment of an invading predatory gastropod on the North American Atlantic Coast. Biological Bulletin. 204: 96-103.

Nyholm N. \& Källqvist T. 1989. Methods for growth inhibition toxicity tests with freshwater algae. Environmental Toxicology and Chemistry. 8: 689703.

OECD (Organization for Economic Cooperation and Development). 1984. Algal growth inhibition test. OECD Guideline for testing Chemicals, No. 201, OECD, Geneva.

Otero A., García D., Morales E.D., Arán J. \& Fábregas J. 1997. Manipulation of the biochemical composition of the eicosapentaenoic acid-rich microalga Isochrysis galbana in semicontinuous cultures. Biotechnology and Applied Biochemical. 26: 171-177.

Pawlisz A.V., Kent R.A., Schneider U.A. \& Jefferson C. 1997. Canadian Water quality Guidelines for chromium. Environmental Toxicology and Water Quality. 12: 185-193.

Pinto E., Sigaud-Kutner T.C.S., Leitao M.A.S., Okamoto O.K., Morse D. \& Colepiloco P. 2003. Heavy metal-induced oxidative stress in algae. Journal of Phycology. 39: 1008-1018.

Podemski C.L. \& Culp J.M. 2001. Toxicant interaction with food algae: A missing link between laboratory and field effects? Environmental Toxicology. 16: 31-42.

Rocchetta I., Ruiz L.B., Magaz G. \& Conforti V.T. 2003. Effects of hexavalent chromium in two strains of Euglena gracilis. Bulletin of Environmental Contamination and Toxicology. 70: 1045-1051.

Silva J., Iannacone J., Cifuentes L., Troncoso L., BaySchmith E. \& Larrain A. 2001. Assessment of sensitivity to Pentachlorophenol (PCP) in 18 aquatic species, using acute and chronic ecotoxicity bioassays. Ecotoxicology and Environmental Restoration. 4: 10-17.

Stauber J.L. \& Florence T.M. 1990. Mechanism of toxicity of zinc to the marine diatom Nitzschia closterium. Marine Biology. 105: 519-524.

USEPA (United States Environmental Protection Agency). 1978. Selenastrum capricornutum Printz algal assay: Bottle test. EPA-600/9-78-018, Corvallis, OR.

Vera G., Tam J., Pinto E. \& Angulo J. 2001. Efecto del cadmio sobre el crecimiento poblacional de la diatomea marina Chaetoceros gracilis Schütt. Revista peruana de Biología. 8: 23-29.

Viamajala S., Peyton B.M., Sani R.K., Apel W.A. \& Petersen J.N. 2004. Toxic effects of chromium (VI) on anaerobic and aerobic growth of Shewanella oneidensis MR-1. Biotechnology Progress. 20: 87-95.

Walsh G.E., Yoder M.J., Mclaughlin L.L. \& Lores E.M. 1987. Response of marine unicellular algae to brominated organic compounds in six growth media. Ecotoxicology and Environmental Safety. 14: 215-222.

Whalley C., Hursthouse A., Rowlatt S., Iqbal-Zahid P., Vaughan H., Durant R.D. 1999. Chromium speciation in natural draining contaminated land, Glasgow, UK. Water, Air and Soil Pollution. 112: 389-405.

Willemsen A., Vaal M.A. \& de Zwart D. 1995. Microbiotests as tools for environmental monitoring. National Institute of Public Health and Environmental Planning (RIVM). The Netherlands, report No 9, 607042005.

Wong P.K. \& Chang L. 1991. Effects of copper, chromium and nickel on the growth, photosynthesis and chlorophyll synthesis of Chlorella pyrenoides. Environmental Pollution. 72: 127-139. 
Villaescusa I., Martí S., Matas C., Martínez M. \& Ribo J.M. 1997. Chromium (VI) toxicity to luminescent bacteria. Environmental Toxicology and Chemistry. 16: 871-874.

Yap C.K., Ismail A., Omar H. \& Tan S.G. 2004. Toxicities and tolerantes of $\mathrm{Cd}, \mathrm{Cu}, \mathrm{Pb}$ and $\mathrm{Zn}$ in as primary producer (Isochrysis galbana) and in a primary consumer (Perna viridis). Environmental International. 29: 1097-1104.
Zayed A.M. \& Terry M. 2003. Chromium in the environment: factors affecting biological remediation. Plant and Soil. 249: 139-156.

Zhu Y., Wang J., Bai Y. \& Zhang R. 2004. Cadmium, chromium, and copper induce polychromatocyte micronuclei in carp (Cyprinus carpio L.). Bulletin Environmental Contamination and Toxicology. 72: 78-86.

\footnotetext{
${ }^{1}$ Laboratorio de Ecofisiología Animal. Facultad de Ciencias Naturales y matemáticas. Universidad Nacional Federico Villarreal. Calle San Marcos 383, Lima - Perú. Correo electrónico: missalayo@hotmail.com

${ }^{2}$ Laboratorio de Ecofisiología Animal. Facultad de Ciencias Naturales y matemáticas. Universidad Nacional Federico Villarreal. Calle San Marcos 383, Lima - Perú. Correo electrónico: joseiannacone@hotmail.com

${ }^{3}$ Laboratorio de Ecofisiología Animal. Facultad de Ciencias Naturales y matemáticas. Universidad Nacional Federico Villarreal. Calle San Marcos 383, Lima - Perú.
} 